\title{
CD10 inhibits cell motility but expression is associated with advanced stage disease in colorectal cancer
}

Teresa P. Raposo a,b* ${ }^{b^{*}}$, Mireia Sueca Comes ${ }^{c}$, Adeyemi Idowu $^{a}$, Bora Agit ${ }^{a}$, James Hassall ${ }^{a, b}$, Wakkas Fadhil ${ }^{a, b}$, Robert Nicad ${ }^{d}$, Rupert Ecker ${ }^{d}$, Takashi Yao ${ }^{e}$, Mohammad llyas ${ }^{a, b}$

a - Division of Cancer and Stem Cells, School of Medicine, University of Nottingham, b- Nottingham Molecular Pathology Node, United Kingdom; c - Biochemistry and Molecular Biology, University Rovira i Virgili, Tarragona, Spain; $d$ - Tissue Gnostics GmbH, Vienna, Austria; e - Department of Human Pathology, Juntendo University, School of Medicine, Tokyo, Japan.

Corresponding author

E-mail address: msztp2@nottingham.ac.uk

Phone number: +441158231395

West block, D floor, Cancer Biology Unit

Division of Cancer and Stem Cells

Queen's Medical Centre

University of Nottingham

Derby Road

Nottingham NG7 2UH

United Kingdom 


\section{Abstract}

Introduction: CD10 is a cell membrane-bound endopeptidase which is expressed in normal small bowel but not in normal colon. It is aberrantly expressed in a small proportion of colorectal cancers (CRC) and this has been associated with liver metastasis and poor prognosis. We sought to investigate the mechanism of CD10 activity and its association with clinicopathological features.

Material and methods: CD10 was stably knocked down by lentiviral shRNA transduction in the CRC cell lines SW480 and SW620 which are derived from a primary tumour and its corresponding metastasis respectively. Expression of epithelial - mesenchymal transition (EMT) markers was tested as well as the effect of knockdown on cell viability, migration and invasion assays. In addition, immunohistochemical expression of CD10 in primary colorectal tumours $(\mathrm{N}=84)$ in a tissue microarray was digitally quantified and analysed for associations with clinicopathological variables.

Results: Knockdown of CD10 did not alter cell viability in SW480, but migration and invasion levels increased ( $P<0.001$ for each) and this was associated with a cadherin switch. In SW620, CD10 knockdown caused a reduction in cell viability after 72 hours $(P=0.0018)$ but it had no effect on cell migration and invasion. Expression of epithelial CD10 in primary tumours was associated with presence of lymph node invasion $(P=0.001)$ and advanced Duke's stage $(\mathrm{P}=0.001)$.

Conclusions: Our results suggest that the function of CD10 may change during tumour evolution. It may inhibit cell motility in early-stage disease whilst promoting cell viability in latestage disease. It has a complex role and further studies are needed to elucidate the suitability of CD10 as a prognostic marker or therapeutic target.

Keywords: CD10, NEP, CALLA, colorectal cancer, epithelial-mesenchymal transition, metastasis

\section{Introduction}

CD10 (cluster of differentiation 10, also designated as neprylisin, membrane metalloendopeptidase (MME), neutral endopeptidase (NEP) and common acute lymphoblastic leukemia antigen (CALLA) is a zinc-dependent metalloendoprotease which degrades peptides involved in cancer signalling pathways (Maguer-Satta et al. 2011). CD10 is expressed in a variety of cells. It is expressed in bone marrow lymphoid stem cells, pro-B lymphoblasts, and mature neutrophils and it is used for classification of leukaemias and malignant lymphomas(Lu 
et al. 2016). It is also seen in the endometrial stroma and, with regards to the epithelium, it is found to be expressed in the lumina of renal tubules, hepatic sinusoids and the microvilli of the small intestine (Lloyd \& Owens 2011; Iwase et al. 2014; Katano et al. 2017).

In solid tumours, CD10 over-expression has found use as a diagnostic marker to distinguish benign from malignant breast phyllodes tumours (Ibrahim 2011; Tariq et al. 2015; Puri et al. 2016) and it has been associated with a poor prognosis in malignant breast cancer (Jana et al. 2014). Similarly, in prostatic cancer cases, CD10 expression was found to correlate with elevated pre-operative prostate-specific antigen (PSA), higher Gleason score and advanced stage (Fleischmann et al. 2008).

CD10 is normally absent in healthy colonic mucosa but it can be aberrantly expressed in both the epithelium and stroma of colorectal tumours and an association with advanced disease is reported (Ogawa et al. 2002). It is not expressed in adenomas with low grade dysplasia but aberrant expression - in both stromal and epithelial compartments - is seen in adenomas with high-grade dysplasia and in invasive adenocarcinomas (Ogawa et al. 2002). CD10 expression has also been associated with increased tumour invasiveness, accumulation of p53 and large tumour size (Ogawa et al. 2002). Moreover, CD10 expression is a significant risk factor for the development of liver metastasis (Fujita et al. 2010; Ohji et al. 2007) and elevated CD10 serum levels have also been shown to be specific predictors of synchronous and metachronous liver metastasis in a cohort of human patients (Sasaki et al. 2014). In a mouse model of metastatic colorectal cancer, CD10 causes degradation of the anti-proliferative protein hepatic metenkephalin thereby enhancing tumour growth (Kuniyasu et al. 2010).

CD10 may also play a role in metastasis in CRC. For metastasis, cells need to undergo epithelial to mesenchymal transition (EMT). During EMT epithelial cells lose their junctions, reorganise their cytoskeleton and undergo a transformational morphological and programming change, which consequently enables an invasive phenotype (Lamouille et al. 2014). Invasive cells may reach blood vessels, circulate to distant organs and settle at the secondary site. For growth at the metastatic site, cells need to undergo the reverse process of mesenchymal to epithelial transition (MET) resulting in formation of metastatic deposits.

We hypothesized that CD10 may have a role in the formation of metastasis through the regulation of the EMT and/or MET processes and thereby altering the motility and invasion of colorectal cancer cells. To test this hypothesis, a stable CD10 knockdown was performed by lentiviral transduction of shRNA in two cell lines obtained from the same patient but derived from the primary tumour and its corresponding metastasis. This allowed us to perform 
functional assays to assess the role of CD10 at the different stages of colorectal cancer in cells with the same genetic background.

\section{Material and methods}

\section{Cell culture}

Colorectal Cancer cell lines SW620 (ATCC® CCL-227 ${ }^{\text {TM}}$ ) and SW480 (ATCC® CCL-228 ${ }^{\text {TM }}$ ) were grown in complete high-glucose DMEM (Dulbecco's Modified Essential Medium; Gibco ${ }^{\circ}$ Life Technologies) supplemented with $2 \mathrm{mM} \mathrm{L-glutamine}$ and $10 \%$ fetal bovine serum (Sigma Aldrich) and incubated at $37^{\circ} \mathrm{C}, 5 \% \mathrm{CO}_{2}$ atmosphere in a humidified incubator.

\section{Lentiviral transduction of shRNA targeting CD10 and Luciferase}

The short hairpin RNA (shRNA) for CD10 and LUC (Luciferase) knockdown were selected using the online tool 'siRNA at Whitehead' (freely available from http://sirna.wi.mit.edu/home.php), using the pattern N2[CG]N8[AUT]N8[AUT]N2 and filtering off-targets with least three mismatches (Pei \& Tuschl 2006).

\begin{tabular}{ll}
\hline & \multicolumn{1}{c}{ shRNA oligo (5'-3') } \\
\hline CD10 Sense & CCGGGCTGGTTGAAACGTAATGTTTCTCGAGTTCGACCAACTTTGCATTACATTTTTG \\
\hline CD10 Antisense & AATTCAAAAAGCTGGTTGAAACGTAATGTTTCTCGAGTTCGACCAACTTTGCATTACA \\
\hline LUC Sense & CCGGGGCGGAAAGTCCAAATTGTTTCTCGAGTTCCGCCTTTCAGGTTTAACATTTTTG \\
\hline LUC Antisense & AATTCAAAAAGGCGGAAAGTCCAAATTGTTTCTCGAGTTCCGCCTTTCAGGTTTAACA \\
\hline Table 1. shRNA oligonucleotides for CD10 and LUC.
\end{tabular}

The shRNA oligos (table 1) were annealed at $95^{\circ} \mathrm{C}$ for 4 minutes in a PCR thermal cycler and slowly cooled down overnight. The annealed oligos were ligated into pLKO.1-TRC (Addgene plasmid \#10878), transformed in chemocompetent E.coli, isolated by miniprep and doubledigested with restriction enzymes Agel and EcoRl. Correctly inserted oligos were confirmed by restriction enzyme double-digestion with EcoRI and Ncol of the resulting vectors and sequencing. Restriction enzymes and chemocompetent $E$. coli were supplied by New England Biolabs.

Viral particles were packaged in HEK293T cells seeded at a density of $1.5 \times 10^{6} / 10 \mathrm{~cm}$ petri dishes in a proportion of $24 \mu \mathrm{L}$ Fugene 6 (Promega, E2691) in 136 $\mu \mathrm{L}$ serum-reduced OPTIMEM (Gibco, Invitrogen) to $2 \mu \mathrm{g}$ pLKO.1 shRNA plasmid: 1500ng psPAX2 packaging plasmid (Addgene \#12260): 500ng pMD2.G envelope plasmid (Addgene \#12259) to 40 $\mu \mathrm{L}$ serumreduced OPTI-MEM. After transfection, shRNA lentiviruses were concentrated by ultracentrifugation at $10,000 \mathrm{xg}, 4^{\circ} \mathrm{C}, 4 \mathrm{~h}$ in a $10 \%$ sucrose buffer (Jiang et al. 2015). 
For stable transfection, SW480 and SW620 cells were pre-treated with $8 \mu \mathrm{g} / \mathrm{mL}$ polybrene (Sigma-Aldrich, \#H9268) for $10 \mathrm{~min}$ and transduced with viral particles containing transfer vectors pLKO.1-TRC-shCD10 or pLKO.1-TRC- shLUC (negative control) for at least 48h. Transduced cells were selected by treatment with puromycin (Sigma-Aldrich, \#P8833) at a pre-tested concentration between $5-10 \mu \mathrm{g} / \mathrm{mL}$ which caused $100 \%$ cell death in untransduced cells. Lentiviral particle production and transduction procedures were adapted from the available protocol supplied by Addgene (Plasmid 10878. Protocol Version 1.0. December 2006), previously used in the preparation of lentiviral shRNA libraries (Moffat et al. 2006).

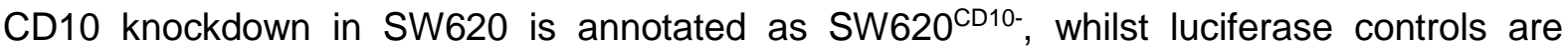
annotated as SW620 LUC- A similar annotation scheme is used for SW480.

\section{Protein extraction and Western blot}

Cells were washed with ice-cold PBS and incubated on ice for 10min with RIPA buffer with protease and phosphatase inhibitor diluted to $1 \mathrm{x}$. The resulting cell lysates were centrifuged at $16000 \mathrm{~g}$, at $4^{\circ} \mathrm{C}$ for $30 \mathrm{~min}$ and the supernatant transferred to a new eppendorf tube. Protein quantification of the supernatant was performed with the Pierce ${ }^{\circledR}$ BCA Assay Kit (Thermo Scientific) according to manufacturer's instructions.

For Western blotting, $50 \mu \mathrm{g}$ of protein sample mixed with $25 \%(\mathrm{v} / \mathrm{v}) \mathrm{NuPAGE}{ }^{\circledR}$ LDS Sample buffer $(4 \mathrm{x})$ and $10 \%(\mathrm{v} / \mathrm{v})$ reducing sample buffer $(10 \mathrm{x})$ were denatured at $95^{\circ} \mathrm{C}$ for $5 \mathrm{~min}$, incubated in ice for 5 minutes and then loaded into to a pre-cast gel (NuPAGE® ${ }^{4-12 \%}$ BisTris Protein Gels, Invitrogen). Gel electrophoresis was performed for $90 \mathrm{~min}$ at $125 \mathrm{~V}$. The resulting gel was immunoblotted onto a polyvinylidene difluoride (PVDF) membrane (Amersham, GE Healthcare Life Sciences) at $25 \mathrm{~V}$ for $30 \mathrm{~min}$, using the BIO-RAD® Trans-Blot Transfer system.

After confirming transfer by Ponceau Red staining, blocking to prevent non-specific protein binding was carried out by incubating the membrane with $5 \%$ skimmed milk (Sigma-Aldrich) or bovine serum albumin (BSA) (ThermoFisher Scientific) in tris-buffered saline $0.1 \%$ Tween20 (TBST) for 1 hour with gentle agitation at room temperature. The membrane was incubated overnight with primary antibody, at $4^{\circ} \mathrm{C}$ with gentle agitation and then was washed $3 \times 5 \mathrm{~min}$ with TBST. It was incubated with the appropriate HRP-conjugated secondary antibody for 1 hour at room temperature, using the blocking solution as the diluent. Diluents and antibodies used are detailed on table A1 (Supplementary data). The membrane was further washed $3 x$ $5 \mathrm{~min}$ in TBST and chemiluminescence was developed using the Amersham ECL Western Blotting Detection Reagent (GE Healthcare Life Sciences). Immunoblots were scanned and analysed by densitometry with the C-DiGit ${ }^{\circ}$ Blot scanner and Image studio system (LI-COR). 
Ratios of Target protein/ $\alpha$-Tubulin intensity ratio and the relative knockdown (KD vs Luciferase control) were calculated (Supplementary figure A1).

\section{Immunofluorescence staining}

SW620 CD10-, SW620 LUC-, SW480 CD10- and SW480 ${ }^{\text {LUC- }}$ were seeded at a density of $2.0 \times 10^{4}$ cells per well of a chamber slide and incubated overnight at $37^{\circ} \mathrm{C}$ in a $5 \% \mathrm{CO}_{2}$ incubator. Cells were washed in PBS (phosphate buffered saline), fixed by incubation in $4 \%$ paraformaldehyde, permeabilized by $0.1 \%$ triton-X 100 PBS and blocked for 1 h with $1 \% \mathrm{BSA}, 0.1 \%$ tween 20 , 22.52mg/mL glycine PBS. CD10 rabbit anti-human antibody diluted 1:100 (Abcam, EPR5904110 ab208778) and alpha-tubulin mouse anti-human diluted 1:200 (Abcam, DM1A ab7291), were incubated overnight at $4^{\circ} \mathrm{C}$, after $3 \times 5$ min washes in $0.1 \%$ Tween 20 in PBS (PBST) secondary antibodies Alexa-Fluor 568 goat anti-rabbit secondary antibody and Alexa-Fluor 488 goat anti-mouse secondary antibody (Invitrogen) were incubated for $1 \mathrm{~h}$, followed by another 3x $5 \mathrm{~min}$ washes in PBST. Nuclei were stained by incubation with DAPI (Sigma Aldrich) diluted 1:5000, for 15min and slides were mounted with fluoroshield anti-fade mountant (Sigma Aldrich). The slides were imaged on a Leica SP2 confocal microscope using the $63 x$ objective with immersion oil.

\section{Cell viability assays and cell cycle analysis}

For cell viability, $1 \times 10^{4}$ cells of each condition of interest (i.e. SW620 CD10-, SW620 LUCSW480 ${ }^{\text {CD10- }}$ and SW480 ${ }^{\text {LUC-) }}$ ) were seeded in each well of a 96 well plate. For each condition, 12 replicate wells were seeded and an individual plate was set up for each of 4 time points. Cells were cultured in a volume of $100 \mu \mathrm{l}$ and incubated at $37^{\circ} \mathrm{C}, 5 \% \mathrm{CO}_{2}$. Cell viability was quantified using a resazurin-based assay with PrestoBlue $\AA^{\circledR}$ Cell Viability Reagent (Invitrogen) diluted to $10 \%$ in cell culture media. To allow correction to the background fluorescence 6 control wells containing only the assay reagent were included. The plate was incubated at $37^{\circ} \mathrm{C}$ for 1 hour and relative fluorescence was read at $544 \mathrm{~nm}$ excitation and $612 \mathrm{~nm}$ emission (FlexStation 384, Molecular Devices). Readings were taken at 0, 24, 48 and 72 hours. To compare between different cell lines, RFU values were normalised to the first performed reading and results expressed as relative cell viability.

For the cell cycle analysis, $1 \times 10^{5}$ cells of each transduced type were seeded into each well of a 24-well plate in quintuplicates and incubated overnight at $37^{\circ} \mathrm{C}, 5 \% \mathrm{CO}_{2}$. A single cell suspension was obtained by trypsinization, washed in PBS and fixed in $70 \%$ cold ethanol for $30 \mathrm{~min}$ at $4^{\circ} \mathrm{C}$, washed twice in PBS and treated with $50 \mu \mathrm{L}$ of a $100 \mu \mathrm{g} / \mathrm{mL}$ stock solution of RNAse (Qiagen). Cells were again re-suspended in $300 \mu \mathrm{L}$ of PBS and nucleic acids stained with $10 \mu \mathrm{L}$ of propidium iodide stock solution at $1 \mathrm{mg} / \mathrm{mL}$ (Sigma Aldrich). Acquisition was 
performed on the FL3 channel of a Cytomics FC500 (Beckman Coulter) and cell cycle parameters analysed on Weasel v 3.0.

\section{Migration and invasion assays}

Cell migration and invasion assays were performed using 24-well polycarbonate transwell membrane inserts (pore size $8 \mu \mathrm{m}$; Corning $囚$ ). For the migration assay, $1 \times 10^{5}$ cells were added to the uncoated transwell membrane inserts in $100 \mu \mathrm{L}$. For the invasion assay, matrigel Matrix (Corning® Matrigel® Matrix) diluted in cold PBS $(100 \mu \mathrm{L}, 300 \mu \mathrm{g} / \mathrm{mL}$ ) was incubated for 2 hours at $37^{\circ} \mathrm{C}$ to coat the transwell insert.

For both the migration and invasion assays, cells were seeded in the top chamber in serumfree DMEM. DMEM with 10\% FBS, $2 \mathrm{mM} \mathrm{L-Glutamine} \mathrm{(650 \mu l)} \mathrm{was} \mathrm{used} \mathrm{as} \mathrm{a} \mathrm{chemoattractant}$ in the receiver well. Quintuplicates of the CD10 knockdown and luciferase control were used for both assays and for each condition wells were established without chemoattractant as a negative control for the motility inducing effects of the serum.

After 24 hours incubation, non-invading cells were removed from the upper chamber. The bottom of the transwells was trypsinized (650 $\mathrm{\mu l}$ trypsin) and trypsinized cells were transferred to the previous receiver well and stained with $4 \mu \mathrm{M}$ of live-cell stain calcein AM (ThermoFisher Scientific) by incubation for $30 \mathrm{~min}$ at $37^{\circ} \mathrm{C}$ under $5 \% \mathrm{CO}_{2}$.

The receiver wells were imaged using an inverted fluorescent microscope at 10x magnification on the FITC channel. To calculate the total number of cells per insert, the mean total number of cells per random microscope field of view $\left(0.055 \mu \mathrm{m}^{2}\right)$ was counted in 4 fields of view and used to estimate the total number of migrated cells present at the bottom of the well $\left(1.9 \mathrm{~cm}^{2}\right)$. Quintuplicates were compared for CD10 knockdown and luciferase control cells. Control wells included no chemoattractant and also no coating for the invasion assay.

\section{Immunohistochemistry (IHC) technique on tissue microarrays (TMAs)}

Immunohistochemical (IHC) staining was performed on a tissue microarray slides (TMA) made from a series of 84 primary colorectal cancers. This series was created specifically for the purpose of evaluating biomarker expression in colorectal tumours and there was an unbiased selection of consecutive cases collected in Nottingham Sciences Biobank at the Queens Medical Centre, Nottingham, UK. Local ethical approval was granted prior to the construction of the TMAs, destined to IHC studies. Formalin-fixed paraffin embedded tissue blocks were retrieved from the archives and a TMA established as previously described (Albasri et al. 2014). 
The TMA set included cores collected from luminal, mid-tumour, advancing edge areas of each tumour and, where possible, adjacent normal areas. Information on clinicopathological parameters tumour grade, primary tumour invasion, vascular invasion, Duke's stage, resection margin, KRAS mutation status and presence of lymph node metastasis was retrieved from histopathology reports preserving the patient anonymity. A summary of the clinicopathological characteristics in the tumour series studied is shown on table B1.

Tissue sections of $4 \mu \mathrm{m}$ thickness were prepared onto poly-L-lysine coated slides (ThermoFisher Scientific, Menz glaser), deparaffinised in xylene, rehydrated by immersion in graded methanol and equilibrated in running tap water for $5 \mathrm{~min}$.

Heat-induced antigen retrieval was performed by immersing the tissue sections in 1mM EDTA buffer $\mathrm{pH} 9.0$ microwaved for $20 \mathrm{~min}$ at $95^{\circ} \mathrm{C}$. After cooling, slides were mounted in Shandon coverplates and sequenza racks (ThermoFisher Scientific) for the remainder IHC incubation steps. For the rest of the procedure, underlined reagents are components of the Novolink Polymer kit (Leica Microsystems) and were used according to manufacturer's instructions. Endogenous peroxidases were blocked using peroxidase block for $5 \mathrm{~min}$. Non-specific antibody binding was blocked by incubation with protein block for $5 \mathrm{~min}$ and washed in TBS (tris-buffered saline).

Each slide was incubated overnight at $4^{\circ} \mathrm{C}$ with rabbit anti-Human CD10 primary antibody (cloneEPR5904-110, ab208778, Abcam) at a 1:2000 dilution. An isotype control anti-rabbit $\lg \mathrm{G}$ was used at the same concentration as the primary antibody. After $3 \times 5 \mathrm{~min}$ washes with TBS, Post-primary reagent was incubated for $30 \mathrm{~min}$ at room temperature, followed by $3 x$ $5 \mathrm{~min}$ TBST washes and incubation with Novolink Polymer for $30 \mathrm{~min}$. Diaminobenzidine (DAB) working solution was applied for $5 \mathrm{~min}$, followed by a final wash in TBS.

Slides were then counterstained using haematoxylin_and excessive stain removed by washes in tap water. After dehydration in methanol and xylene sections were mounted using mounting medium (DPX). Finally, slides were digitalised using a nanozoomer scanner (Hammamatsu).

Digital image analysis was performed on the software Strataquest v.6.0 (Tissue Gnostics). After colour separation and classifier-based tumour epithelium-stroma segmentation, ratios (\%) were calculated for the CD10 positive epithelial area in the total tumour epithelium and CD10 positive stromal area within the total tumour stroma of each individual core. Misidentified epithelial and stromal areas were manually corrected if needed after individual verification of the segmentation results. The values of CD10 positive epithelium ratios for 
luminal, mid-tumour and advancing edge cores were added to calculate the total CD10 epithelium score.

\section{Statistical analysis}

GraphPad Prism 7.01 (CA, USA) was used for statistical analysis of in vitro assays. Two-way analysis of variance (ANOVA) was performed to analyse proliferation assays and cell cycle analysis. Unpaired Mann-Whitney T-test (non-parametric test) was performed to compare means in protein expression, migration and invasion assay results. The statistical software SPSS (Statistical Package for the Social Sciences, IBM, OH, USA) version 22.0 was used for IHC statistical analysis of associations with clinicopathological variables by Pearson Chisquare test. The normality test Kolmogorov-Smirnov was firstly applied to confirm the population followed a non-Gaussian distribution. Cut off values were determined by the median value of the total epithelial or stromal CD10 score. In all statistical comparisons, $p<0.05$ was accepted as denoting significance difference.

\section{Results}

\section{Confirmation of CD10 knockdown by Western Blot and Immunofluorescence}

After transduction with lentiviruses expressing shCD10 or shLUC oligos, the protein level was measured by Western Blot. CD10 protein expression was visibly decreased for both transduced shC10 cell lines (SW480 ${ }^{\mathrm{CD} 10-}$ and SW620 ${ }^{\mathrm{CD} 10-}$, Figure 1). These changes were also visible by immunofluorescence staining of CD10, where the fusiform morphology acquired by SW480 ${ }^{\mathrm{CD} 10-}$ could also be observed (Figure 2).

\section{Effect of CD10 knockdown on cell viability}

Cell viability was assessed at over a time course of 72 hours. In SW480 CD10- cell viability was not altered as growth curves of the cells were identical to SW480 Luc (Figure $3 \mathrm{~A}$ ). In contrast, in SW620 ${ }^{\mathrm{CD} 10-}$ a delayed inhibition of cell growth which only became apparent after 72 hours (Figure 3B and B1). A cell cycle analysis confirmed a small but significant decrease in the G2/M phase post CD10 knockdown in SW620 ${ }^{C D 10-}$ and SW480 ${ }^{C D 10-}(P=0.0143$ and $P=0.0059$ respectively, Figure $2 \mathrm{C}$ ).

\section{Effect of CD10 knockdown on cell migration and invasion}


Since no difference was observed in the effect of CD10 knockdown on the proliferative activity at $24 \mathrm{~h}$, there was no need to block cell replication before performing migration and invasion assays (which are conducted in 24h). In SW480 ${ }^{\text {CD10- }}$ cells, the knockdown of CD10 resulted in an increase in cell motility. A statistically significant increase in cell numbers was observed in both the transwell migration and invasion assays when compared to the control cell line $(P<0.001)$ (Figure 3, C and D). In contrast, in SW620 CD10-, no significant differences were observed in either transwell migration or invasion assays between CD10 knockdown and luciferase control (Figure 3, A and B).

\section{Identification of CD10-associated EMT Markers}

Expression of a variety of biomarkers related to the EMT process was assessed by Western blot. E-Cadherin and N-Cadherin are cell adhesion molecules and ordinarily, in the non-motile state, E-cadherin levels are high whilst N-Cadherin levels are low. When cells become motile, the levels are reversed with high $\mathrm{N}$-Cadherin and low E-cadherin (known as the "cadherin switch" (Maeda et al. 2005). CD10 knockdown in SW480 resulted in a cadherin switch resulting in reduced $\mathrm{E}$-Cadherin expression and increased $\mathrm{N}$-Cadherin expression (Figure 1 , Supplementary Figure 1) thus complementing the observed changes in cell motility. Evaluation of other EMT markers showed that, despite the cadherin switch and increased motility, Snail and Twist were reduced and Vimentin remained unchanged. However, these changes were not seen with CD10 knockdown in SW620 - there was no cadherin switch and the expression of Snail, Twist and vimentin was unchanged. CD10 Knockdown was associated with slight increase on $\mathrm{N}$-Cadherin and $\mathrm{E}$-Cadherin expression (Figure 1). Densitometry plots are shown on figure A1.

\section{CD10 expression in primary colorectal tumours}

Immunohistochemistry for expression of CD10 was performed on a TMA containing 84 primary colorectal cancers. Expression was seen both within the tumour epithelium and the stroma. Within the epithelium there was a mixture of membranous and cytoplasmic expression with varying levels of expression seen. The stromal cells generally showed weaker expression than the epithelium and mostly within the cytoplasm. Representative areas of high or low epithelial and stromal ratio of CD10 immunohistochemical staining are shown on Figure 5.

Since both stromal and epithelial areas show staining for CD10, automated image analysis software was used to segment out and quantify the level of expression in each compartment. In addition, since cores were taken from 4 different regions (luminal area, mid-tumour, advancing edge and adjacent normal area), each region was examined individually. For an overall evaluation of expression, a total epithelial and stromal score was calculated from the 
sum of the ratios at the luminal, mid-tumour and advancing edge. To obtain a grade (low or high) of CD10 expression, the epithelial and stromal scores were split in two categories by the median value.

Statistically significant associations were detected between high epithelial CD10 expression and presence of lymph node metastasis $(P=0.001)$ and advanced Duke's stage $(P=0.001)$. A high stromal CD10 expression was also associated with KRAS wild-type status $(P=0.043)$ (Table 2).

Non-parametric Mann-Whitney ranks comparison of the four Duke's stage groups analysed demonstrates statistically significant differences in epithelial CD10 expression are located between Duke's stage A and C1 $(P=0.012)$ and between stage $B$ and C1 $(P=0.029)$ (Figure $6)$.

\begin{tabular}{|c|c|c|c|c|c|c|}
\hline & \multicolumn{3}{|c|}{ Epithelial CD10 score } & \multicolumn{3}{|c|}{ Stromal CD10 score } \\
\hline & Low & High & $\begin{array}{l}\text { P- } \\
\text { value }\end{array}$ & Low & High & P-value \\
\hline $\begin{array}{l}\text { Grade } \\
1 \\
2 \\
3\end{array}$ & $\begin{array}{l}1 \\
33 \\
4\end{array}$ & $\begin{array}{l}1 \\
35 \\
1\end{array}$ & 0.397 & $\begin{array}{l}1 \\
35 \\
1\end{array}$ & $\begin{array}{l}1 \\
32 \\
4\end{array}$ & 0.380 \\
\hline $\begin{array}{l}\text { Tumour invasiveness }{ }^{a} \\
1 \text { or } 2 \\
3 \text { or } 4\end{array}$ & $\begin{array}{l}9 \\
29\end{array}$ & $\begin{array}{l}5 \\
32\end{array}$ & 0.258 & $\begin{array}{l}5 \\
32\end{array}$ & $\begin{array}{l}9 \\
28\end{array}$ & 0.235 \\
\hline $\begin{array}{l}\text { Duke's stage } \\
A \text { and } B \\
C 1 \text { and } C 2\end{array}$ & $\begin{array}{l}30 \\
16\end{array}$ & $\begin{array}{l}7 \\
21\end{array}$ & 0.001 & $\begin{array}{l}24 \\
12\end{array}$ & $\begin{array}{l}24 \\
13\end{array}$ & 0.871 \\
\hline $\begin{array}{l}\text { Ressection margin } \\
\text { Free } \\
\text { Involved }\end{array}$ & $\begin{array}{l}35 \\
1\end{array}$ & $\begin{array}{l}35 \\
2\end{array}$ & 0.572 & $\begin{array}{l}33 \\
2\end{array}$ & $\begin{array}{l}35 \\
2\end{array}$ & 0.954 \\
\hline $\begin{array}{l}\text { Vascular invasion } \\
\text { Present } \\
\text { Absent }\end{array}$ & $\begin{array}{l}15 \\
23\end{array}$ & $\begin{array}{l}22 \\
15\end{array}$ & 0.083 & $\begin{array}{l}18 \\
19\end{array}$ & $\begin{array}{l}16 \\
21\end{array}$ & 0.641 \\
\hline $\begin{array}{l}\text { LN metastasis }{ }^{b} \\
\text { Present } \\
\text { Absent }\end{array}$ & $\begin{array}{l}17 \\
31\end{array}$ & $\begin{array}{l}20 \\
7\end{array}$ & 0.001 & $\begin{array}{l}26 \\
11\end{array}$ & $\begin{array}{l}11 \\
13\end{array}$ & 0.619 \\
\hline $\begin{array}{l}\text { KRAS status }^{\mathrm{b}} \\
\text { Wild-type } \\
\text { Mutant }\end{array}$ & $\begin{array}{l}21 \\
21\end{array}$ & $\begin{array}{l}13 \\
11\end{array}$ & 0.745 & $\begin{array}{l}14 \\
16\end{array}$ & $\begin{array}{l}26 \\
10\end{array}$ & 0.034 \\
\hline
\end{tabular}

Table 2 - Associations between clinicopathological variables and the epitehlial and stromal CD10 expression ratios. Pearson Chi-square was used to compare the CD10 high or low total epithelial and stromal scores for each of the categories considered. P-values $<0.05$ are considered statistically significant and are highlighted in bold.

a - Tumour invasiveness categories grouping corresponds to: Submucosa invasion (1); Muscularis propria invasion (2); Muscularis and pericolorectal invasion (3); Visceral peritoneum and organs (4).

\section{Discussion}

In this study we have examined the role of tumour-associated CD10 in the CRC biology by comparing proliferation, migration and invasion of an isogenic pair of colorectal cancer cell 
lines derived from different tumour sites (i.e. SW480 was derived from the primary tumour whilst SW620 was derived from a metastatic deposit of the same tumour).

CD10 knockdown was achieved by stable lentiviral transduction with PLKO.1-shCD10, confirmed by western blot (Figure 1). Following stable CD10 knockdown, a reduction in cell viability in SW620 ${ }^{\mathrm{CD} 10-}$ was observed albeit only after 72 hours in culture (Figure 2B). However, in SW480 ${ }^{\mathrm{CD} 10-}$, there was no significant difference in cell viability (Figure 2A). Our results can be compared to those obtained by Mizerska-Kowalska et al, who have also observed a reduced proliferation (determined by the BrdU method) and increased proportion of apoptotic cells following CD10 knockdown in the CRC cell line LS180 (Mizerska-Kowalska et al. 2016). In their case however, cell cycle analysis showed G2/M arrest upon transient CD10 knockdown in LS180 cells but not in SW620 (Mizerska-Kowalska et al. 2016). In contrast, although we have observed the same net effect on proliferation following CD10 knockdown, we have obtained a small but significant decrease in G2/M. These small shifts in proliferation could be due to a reduction of focal adhesion kinase phosphorylation at Tyr 397, regulated by CD10 intrinsic signalling (Mizerska-Kowalska et al. 2016). In terms of cell viability and proliferation, we must recognize the nature of the effects of the tumour microenvironment at the primary tumour (SW480) and metastatic site (SW620) and its long term implications in the metabolic activity of the cell lines hereby studied.

Considering the proteolytic function of CD10 and previous associations with cancer progression and metastasis, we had expected that, in line with published studies (Fujimoto et al. 2005; Kuniyasu et al. 2010; Iwase et al. 2005; Jang et al. 2013; Fujita et al. 2010; Ohji et al. 2007), CD10 silencing would cause a reduction in migration and invasiveness of colorectal cancer cell lines. However our data showed that CD10 silencing resulted in increased migration and invasion of SW480 ${ }^{\mathrm{CD} 10-}$ cell lines, while in SW620 ${ }^{\mathrm{CD} 10-}$ there was no significant change (Figure 4). Comparable results were obtained by Mizerska-Kowalska et al. who showed that CD10 silencing caused an increase in invasion and migration in LS180. In SW620, they found that CD10 silencing caused increased migration but reduced invasion (Mizerska-Kowalska et al. 2016). This also suggests that CD10 can inhibit cell migration and was attributed to the observation that CD10 silencing induced FAK phosphorylation thereby enhancing cell motility (Mizerska-Kowalska et al. 2016). The reason for the differences in the results in SW620 between our study and that of Mizerska-Kowalska is uncertain but, taking all the data together, there is evidence to suggest that, CD10 can inhibit cell migration.

Knockdown of CD10 in SW480 caused a cadherin shift marked by a decrease in E-cadherin, coupled with an increase in $\mathrm{N}$-cadherin expression (Figure 1). These results complement our observed differences for migration and invasion. Reduction of E-cadherin, often the result of 
EMT, is a frequent event during tumour progression and metastasis (Polyak \& Weinberg 2009). Reduced E-cadherin is often accompanied by an increase in N-cadherin during a 'cadherin switch' which results in increased cell motility (Maeda et al. 2005). Unexpectedly, the cadherin switch occurred simultaneously with downregulation of Vimentin, Snail and Twist - markers characteristically associated with EMT. This downregulation would usually be associated with reduced motility. The data are suggestive of the plasticity of the mechanisms involved in processes of cell motility and EMT (Huang et al. 2013; Grigore et al. 2016). Loss of E-cadherin expression, with maintenance of high $\mathrm{N}$-cadherin levels is likely to alter the adhesiveness of cells (Chu et al. 2006), and thus may explain the induction of motility without acquisition of a complete EMT shift in SW480 ${ }^{\mathrm{CD} 10}$. The precise mechanism by which CD10 could be involved in regulating the EMT process by $\mathrm{E}$-cadherin and $\mathrm{N}$-cadherin deserves further investigation.

Published data are inconsistent when IHC for CD10 is evaluated. Some studies have shown high CD10 expression in well and moderately-differentiated colon carcinomas relative to poorly differentiated ones (Sato et al. 1996; Fujimoto et al. 2005). Others have found a progressive increase in CD10 expression from normal to benign tumours to invasive disease (Jang et al. 2013; Yao et al. 2002). In our case only malignant colorectal adenocarcinomas were analysed, and therefore a stepwise association with CD10 expression in normal, benign and malignant tumours could not be made.

While most studies on CD10 have used a positive/negative categorical or semi-quantitative evaluation, we have employed an automated IHC quantification method that excludes the observer-associated subjectivity. When expression of CD10 in the epithelial and stromal tumour compartments was analysed for its associations with clinicopathological variables, high CD10 expression in tumour epithelial cells was associate with advance disease (Dukes' $A \& B$ versus Dukes' $C 1$ and $C 2, P=0.001)$. Differences in $C D 10$ expression were particularly evident in a pairwise ranks comparison between Duke's stage $A$ and $C 1(P=0.012)$ and $B$ and C1 $(P=0.029)$. Stromal CD10 expression was not associated to Duke's stage. Similarly, epithelial CD10 expression was associated with presence of lymph node metastasis $(P<0.001)$. While this is not completely in agreement with our in vitro results showing CD10 as an inhibitor of migration and invasion in SW480, in vivo epithelial CD10 expression could be counteracting anti-tumoural effect of peptides such as met-enkephalin, and as a consequence, promoting invasion and lymph node metastasis (Kuniyasu et al. 2010). It has also been shown that CD10 enzymatic activity degrades migration-inducing peptides such as bombesin and endothelin (Terauchi et al. 2005; Zheng et al. 2006; Wang et al. 2017). It is therefore feasible that CD10 activitiy in vivo is conditioned by the relative abundance of such peptides in different 
tumour compartments and tumour stages, explaining discrepancies to data obtained in vitro where extrinsic peptidase activity of CD10 could not be assessed.

\section{Conclusion}

Considering the results hereby presented, it is possible that proteolytic effects of CD10expression are controlled by the surrounding microenvironment and its significance depends on the tumour stage and interaction of stroma and epithelium. Our findings suggest that CD10 knockdown has the potential to increase the migrating and invading ability of the primary colorectal cancer cell line SW480, but not that of the matched metastasis SW620. Intriguingly, results of our TMA analysis on CD10 immunostaining show and association with advanced Duke's stage and presence of lymph node metastasis.

In vitro studies which do not fully recapitulate the rich microenvironment displayed within colorectal tumour could nevertheless suggest alterations in proliferation, migration and invasion together with shifts in EMT markers caused by CD10 knockdown, all of which seem to be influenced by the stage of tumour development. Due to the importance of understanding mechanisms underlying tumour invasion and metastasis, further research is warranted to clarify the role of CD10 in EMT and tumour progression.

\section{Acknowledgements}

The authors acknowledge financial support from the EC Marie Curie Actions, AIDPATH project (Contract No.612471). Support was also provided by an International Collaborator Award from the Pathological Society of Great Britain and Ireland. Takashi Yao received financial support from Grant-in-Aid for General Scientific Research from the Ministry of Education, Science, Sports and Culture (\#17K08704), Japan.

\section{Conflict of interest}

Takashi Yao received honoraria for lectures from Takeda Pharmaceutical Co. All other authors declare no conflict of interest. 


\section{References}

Albasri, A. et al., 2014. Nuclear expression of phosphorylated focal adhesion kinase is associated with poor prognosis in human colorectal cancer. Anticancer research, 34(8), pp.3969-74.

Chu, Y.-S. et al., 2006. Prototypical Type I E-cadherin and Type II Cadherin-7 Mediate Very Distinct Adhesiveness through Their Extracellular Domains. Journal of Biological Chemistry, 281(5), pp.2901-2910.

Fleischmann, A. et al., 2008. Distinct Subcellular Expression Patterns of Neutral Endopeptidase (CD10) in Prostate Cancer Predict Diverging Clinical Courses in Surgically Treated Patients. Clinical Cancer Research, 14(23), pp.7838-7842.

Fujimoto, Y. et al., 2005. CD10 Expression in Colorectal Carcinoma Correlates With Liver Metastasis. Diseases of the Colon \& Rectum, 48(10), pp.1883-1889.

Fujita, S. et al., 2010. Multi-institutional study of risk factors of liver metastasis from colorectal cancer: correlation with CD10 expression. International journal of colorectal disease, 25(6), pp.681-6.

Grigore, A. et al., 2016. Tumor Budding: The Name is EMT. Partial EMT. Journal of Clinical Medicine, 5(5), p.51.

Huang, R.Y.-J. et al., 2013. An EMT spectrum defines an anoikis-resistant and spheroidogenic intermediate mesenchymal state that is sensitive to e-cadherin restoration by a srckinase inhibitor, saracatinib (AZD0530). Cell Death and Disease, 4(11), p.e915.

Ibrahim, W.S., 2011. Comparison of stromal CD10 expression in benign, borderline, and malignant phyllodes tumors among Egyptian female patients. Indian journal of pathology \& microbiology, 54(4), pp.741-4.

Iwase, A. et al., 2014. Possible Involvement of CD10 in the Development of Endometriosis Due to Its Inhibitory Effects on CD44-Dependent Cell Adhesion. Reproductive Sciences, 21(1), pp.82-88.

Iwase, T. et al., 2005. Overexpression of CD10 and reduced MUC2 expression correlate with the development and progression of colorectal neoplasms. Pathology - Research and Practice, 201(2), pp.83-91. 
Jana, S. et al., 2014. CD10-A new prognostic stromal marker in breast carcinoma, its utility, limitations and role in breast cancer pathogenesis. Indian Journal of Pathology and Microbiology, 57(4), p.530.

Jang, T.J., Park, J.B. \& Lee, J.I., 2013. The Expression of CD10 and CD15 Is Progressively Increased during Colorectal Cancer Development. Korean journal of pathology, 47(4), pp.340-7.

Jiang, W. et al., 2015. An optimized method for high-titer lentivirus preparations without ultracentrifugation. Scientific Reports, 5, p.13875.

Katano, T. et al., 2017. Ectopic Gastric and Intestinal Phenotypes, Neuroendocrine Cell Differentiation, and SOX2 Expression Correlated With Early Tumor Progression in Colorectal Laterally Spreading Tumors. Clinical Colorectal Cancer, 16(2), pp.141-146.

Kuniyasu, H. et al., 2010. CD10 enhances metastasis of colorectal cancer by abrogating the anti-tumoural effect of methionine-enkephalin in the liver. Gut, 59(3), pp.348-56.

Lamouille, S., Xu, J. \& Derynck, R., 2014. Molecular mechanisms of epithelial-mesenchymal transition. Nature reviews. Molecular cell biology, 15(3), pp.178-96.

Lloyd, J.M. \& Owens, S.R., 2011. CD10 immunohistochemistry stains enteric mucosa, but negative staining is unreliable in the setting of active enteritis. Modern Pathology, 24(12), pp.1627-1632.

Lu, T.-X. et al., 2016. The distinct clinical features and prognosis of the CD10+MUM1+ and CD10-Bcl6-MUM1- diffuse large B-cell lymphoma. Scientific Reports, 6(1), p.20465.

Maeda, M., Johnson, K.R. \& Wheelock, M.J., 2005. Cadherin switching: essential for behavioral but not morphological changes during an epithelium-to-mesenchyme transition. Journal of Cell Science, 118(5), pp.873-887.

Maguer-Satta, V., Besançon, R. \& Bachelard-Cascales, E., 2011. Concise Review: Neutral Endopeptidase (CD10): A Multifaceted Environment Actor in Stem Cells, Physiological Mechanisms, and Cancer. STEM CELLS, 29(3), pp.389-396.

Mizerska-Kowalska, M. et al., 2016. Neutral endopeptidase (NEP) is differentially involved in biological activities and cell signaling of colon cancer cell lines derived from various stages of tumor development. Tumor Biology, 37(10), pp.13355-13368.

Moffat, J. et al., 2006. A lentiviral RNAi library for human and mouse genes applied to an 
arrayed viral high-content screen. Cell, 124(6), pp.1283-98.

Ogawa, H. et al., 2002. Expression of CD10 by stromal cells during colorectal tumor development. Human pathology, 33(8), pp.806-11.

Ohji, Y. et al., 2007. Evaluation of risk of liver metastasis in colorectal adenocarcinoma based on the combination of risk factors including CD10 expression: multivariate analysis of clinicopathological and immunohistochemical factors. Oncology reports, 17(3), pp.52530.

Pei, Y. \& Tuschl, T., 2006. On the art of identifying effective and specific siRNAs. Nature Methods, 3(9), pp.670-676.

Polyak, K. \& Weinberg, R.A., 2009. Transitions between epithelial and mesenchymal states: acquisition of malignant and stem cell traits. Nature Reviews Cancer, 9(4), pp.265-273.

Puri, V. et al., 2016. Critical appraisal of stromal CD10 staining in fibroepithelial lesions of breast with a special emphasis on expression patterns and correlation with WHO grading. Journal of cancer research and therapeutics, 12(2), pp.667-70.

Sasaki, T. et al., 2014. Serum CD10 is associated with liver metastasis in colorectal cancer. The Journal of surgical research, 192(2), pp.390-4.

Sato, Y. et al., 1996. Expression of CD10/neutral endopeptidase in normal and malignant tissues of the human stomach and colon. Journal of Gastroenterology, 31(1), pp.12-17.

Tariq, M.U., Haroon, S. \& Kayani, N., 2015. Role of CD10 Immunohistochemical Expression in Predicting Aggressive Behavior of Phylloides Tumors. Asian Pacific journal of cancer prevention : APJCP, 16(8), pp.3147-52.

Terauchi, M. et al., 2005. Anti-Progressive Effect of Neutral Endopeptidase 24.11 (NEP/CD10) on Cervical Carcinoma in vitro and in vivo. Oncology, 69(1), pp.52-62.

Wang, Z. et al., 2017. Endothelin Promotes Colorectal Tumorigenesis by Activating YAP/TAZ. Cancer Research, 77(9), pp.2413-2423.

Yao, T. et al., 2002. Phenotypic expression of gastrointestinal differentiation markers in colorectal adenocarcinomas with liver metastasis. Pathology, 34(6), pp.556-60.

Zheng, R. et al., 2006. Neuropeptide-stimulated cell migration in prostate cancer cells is mediated by RhoA kinase signaling and inhibited by neutral endopeptidase. Oncogene, 
25(44), pp.5942-5952. 
Appendix: Supplementary tables

\begin{tabular}{|c|c|c|c|c|c|}
\hline \multicolumn{2}{|c|}{ Primary antibody } & \multirow{2}{*}{$\begin{array}{l}\text { Diluent } \\
5 \% \\
\text { skimmed } \\
\text { milk, } \\
\text { TBST }\end{array}$} & \multirow{2}{*}{$\begin{array}{l}\text { Dilution } \\
1: 500\end{array}$} & \multirow{2}{*}{$\begin{array}{l}\text { Secondary } \\
\text { antibody } \\
\text { Goat } \alpha \text {-Rabbit } \\
\text { (Sigma Aldrich } \\
\text { A6154)... }\end{array}$} & \multirow{2}{*}{$\begin{array}{l}\text { Dilution } \\
1: 1000\end{array}$} \\
\hline CD10 & $\begin{array}{l}\text { Rabbit } \alpha \text {-Human } \\
\text { (Abcam, } \\
\text { EPR5904-110 } \\
\text { ab208778) }\end{array}$ & & & & \\
\hline N-Cadherin & $\begin{array}{l}\text { Rabbit } \alpha \text {-Human } \\
\text { (Abcam, ab12221) }\end{array}$ & $\begin{array}{l}5 \% \\
\text { skimmed } \\
\text { milk, } \\
\text { TBST }\end{array}$ & $1: 1000$ & $\begin{array}{l}\text { Goat } \alpha \text {-Rabbit } \\
\text { (Sigma Aldrich } \\
\text { A6154) }\end{array}$ & $1: 1000$ \\
\hline E-Cadherin & $\begin{array}{l}\text { Rabbit } \alpha \text {-Human } \\
\text { (CST, \#3195) }\end{array}$ & $\begin{array}{l}5 \% \text { BSA, } \\
\text { TBST }\end{array}$ & $1: 1000$ & $\begin{array}{l}\text { Goat } \alpha \text {-Rabbit } \\
\text { (Sigma Aldrich } \\
\text { A6154) }\end{array}$ & $1: 1000$ \\
\hline Snail & $\begin{array}{l}\text { Rabbit } \alpha \text {-Human } \\
\text { (CST, \#3879S) }\end{array}$ & $\begin{array}{l}5 \% \text { BSA, } \\
\text { TBST }\end{array}$ & $1: 1000$ & $\begin{array}{l}\text { Goat } \alpha \text {-Rabbit } \\
\text { (Sigma Aldrich } \\
\text { A6154) }\end{array}$ & $1: 1000$ \\
\hline Vimentin & $\begin{array}{l}\text { Rabbit } \alpha \text {-Human } \\
\text { (CST, \#5741S) }\end{array}$ & $\begin{array}{l}5 \% \text { BSA, } \\
\text { TBST }\end{array}$ & $1: 1000$ & $\begin{array}{l}\text { Goat } \alpha \text {-Rabbit } \\
\text { (Sigma Aldrich } \\
\text { A6154) }\end{array}$ & $1: 1000$ \\
\hline Twist & $\begin{array}{l}\text { Mouse } \alpha \text {-Human } \\
\text { (Abcam, } \\
\text { ab175430) }\end{array}$ & $\begin{array}{l}5 \% \text { BSA, } \\
\text { TBST }\end{array}$ & $1: 1000$ & $\begin{array}{l}\text { Rabbit } \alpha \text {-Mouse } \\
\text { (Sigma Aldrich, } \\
\text { A4416) }\end{array}$ & $1: 1000$ \\
\hline$\alpha$-Tubulin & $\begin{array}{l}\text { Mouse } \alpha \text {-Human } \\
\text { (Abcam, DM1A } \\
\text { ab7291) }\end{array}$ & $\begin{array}{l}5 \% \\
\text { skimmed } \\
\text { milk, } \\
\text { TBST }\end{array}$ & $1: 2000$ & $\begin{array}{l}\text { Rabbit } \alpha \text {-Mouse } \\
\text { (Sigma Aldrich, } \\
\text { A4416) }\end{array}$ & $1: 5000$ \\
\hline
\end{tabular}

Table A1-Dilution ratios for primary and secondary antibodies and diluent used for each antibody. 


\begin{tabular}{|c|c|c|}
\hline & Frequency(N) & $(\%)$ \\
\hline \multicolumn{3}{|l|}{ Resection type ( $\mathrm{N}=81$ ) } \\
\hline Anterior resection & 23 & 27.4 \\
\hline Aper & 2 & 2.4 \\
\hline Caecum resection & 1 & 1.2 \\
\hline Hartmanns & 1 & 1.2 \\
\hline Left hemicolectomy & 1 & 1.2 \\
\hline Rectum resection & 1 & 1.2 \\
\hline Right hemicoloctomy & 33 & 39.3 \\
\hline Sigmoid colectomy & 16 & 19.0 \\
\hline Small intestine resection & 1 & 1.2 \\
\hline Total colectomy & 1 & 1.2 \\
\hline Transverse colon resection & 1 & 1.2 \\
\hline \multicolumn{3}{|l|}{ Tumour grade $(\mathrm{N}=84)$} \\
\hline 1 & 2 & 2.4 \\
\hline 2 & 77 & 91.7 \\
\hline 3 & 5 & 6.0 \\
\hline \multicolumn{3}{|l|}{ Invasiveness ( $\mathrm{N}=84)$} \\
\hline Submucosa invasion (1) & 3 & 3.6 \\
\hline $\begin{array}{l}\text { Muscularis propria invasion } \\
\text { (2) }\end{array}$ & 12 & 14.3 \\
\hline $\begin{array}{l}\text { Muscularis and pericolorectal } \\
\text { invasion (3) }\end{array}$ & 46 & 54.8 \\
\hline $\begin{array}{l}\text { Visceral peritoneum and } \\
\text { organs (4) }\end{array}$ & 23 & 27.4 \\
\hline \multicolumn{3}{|l|}{ Resection margin $(\mathrm{N}=82)$} \\
\hline Free & 78 & 95.1 \\
\hline Involved & 4 & 4.9 \\
\hline \multicolumn{3}{|l|}{ Vascular invasion $(\mathrm{N}=84)$} \\
\hline Absent & 42 & 50.0 \\
\hline Present & 42 & 50.0 \\
\hline \multicolumn{3}{|l|}{ Duke's stage $(\mathrm{N}=83)$} \\
\hline$A$ & 13 & 15.5 \\
\hline B & 38 & 45.2 \\
\hline C1 & 28 & 33.3 \\
\hline $\mathrm{C} 2$ & 4 & 4.8 \\
\hline \multicolumn{3}{|l|}{$\begin{array}{l}\text { KRAS } \\
(\mathrm{N}=75)\end{array}$ mutation $\quad$ status } \\
\hline Wild-type & 46 & 54.8 \\
\hline Mutant & 29 & 34.5 \\
\hline \multicolumn{3}{|l|}{ LN Metastasis $(\mathrm{N}=84)$} \\
\hline Absent & 53 & 63.1 \\
\hline Present & 31 & 36.9 \\
\hline
\end{tabular}

Table B1- Summary of clinicopathological characteristics in the patient samples included in this study. 